\title{
Furin Cleavage Site Is Key to SARS-CoV-2 Pathogenesis
}

Bryan A. Johnson ${ }^{1^{\star}}$, Xuping Xie ${ }^{2^{*}}$, Birte Kalveram ${ }^{3}$, Kumari G. Lokugamage ${ }^{1}$, Antonio Muruato ${ }^{1}$, Jing Zou ${ }^{2}$, Xianwen Zhang ${ }^{2}$, Terry Juelich ${ }^{3}$, Jennifer K. Smith ${ }^{3}$, Lihong Zhang ${ }^{3}$, Nathen Bopp ${ }^{3}$, Craig Schindewolf ${ }^{1}$, Michelle Vu ${ }^{1}$, Abigail Vanderheiden ${ }^{5,6,7}$, Daniele Swetnam ${ }^{2}$, Jessica A. Plante $^{1}$, Patricia Aguilar ${ }^{3}$, Kenneth S. Plante ${ }^{1}$, Benhur Lee ${ }^{8}$, Scott C. Weaver $^{1,4}$, Mehul S. Suthar ${ }^{5,6,7}$, Andrew L. Routh ${ }^{2}$, Ping $\mathrm{Ren}^{3}$, Zhiqiang $\mathrm{Ku}^{9}$, Zhiqiang $\mathrm{An}^{9}$, Kari Debbink ${ }^{10}$, Pei Yong $\mathrm{Shi}^{2,4, \#}$, Alexander N. Freiberg ${ }^{3,4, \#}$, Vineet D. Menachery ${ }^{1,4, \#}$

${ }^{1}$ Departments of Microbiology and Immunology, ${ }^{2}$ Biochemistry and Molecular Biology,

${ }^{3}$ Pathology, ${ }^{4}$ Institute for Human Infection and Immunity, University of Texas Medical Branch, Galveston, TX, USA

${ }^{5}$ Department of Pediatrics, ${ }^{6}$ Emory Vaccine Center, Emory University School of Medicine, Atlanta, GA, USA.

${ }^{7}$ Yerkes National Primate Research Center, Atlanta, GA, USA

${ }^{8}$ Icahn School of Medicine at Mount Sinai, New York, NY, USA

${ }^{9}$ Texas Therapeutics Institute, Brown Foundation Institute of Molecular Medicine, University of Texas Health Science Center at Houston, Houston, USA.

${ }^{10}$ Bowie State University, Bowie, MD, USA

*Equal contributions

${ }^{\#}$ Co-senior authors

Corresponding Author: Vineet D. Menachery

Address: University of Texas Medical Branch, 301 University Blvd, Route \#0610 Galveston, TX 77555

Email: Vimenach@utmb.edu

Article Summary: A deletion of the furin cleavage site in SARS-CoV-2 amplifies replication in Vero cells, but attenuates replication in respiratory cells and pathogenesis in vivo. Loss of the furin site also reduces susceptibility to neutralization in vitro.

Running title: Furin cleavage site is key to SARS-CoV-2 replication and pathogenesis.

Keywords: Coronavirus, 2019-nCoV, SARS-CoV-2, COVID-19, furin-cleavage, spike 


\section{$1 \quad$ Abstract}

2 SARS-CoV-2 has resulted in a global pandemic and shutdown economies around the world.

3 Sequence analysis indicates that the novel coronavirus (CoV) has an insertion of a furin

4 cleavage site (PRRAR) in its spike protein. Absent in other group 2B CoVs, the insertion may

5 be a key factor in the replication and virulence of SARS-CoV-2. To explore this question, we generated a SARS-CoV-2 mutant lacking the furin cleavage site ( $\triangle \mathrm{PRRA})$ in the spike protein.

7 This mutant virus replicated with faster kinetics and improved fitness in Vero E6 cells. The mutant virus also had reduced spike protein processing as compared to wild-type SARS-CoV-2.

9 In contrast, the $\triangle$ PRRA had reduced replication in Calu3 cells, a human respiratory cell line, and

10 had attenuated disease in a hamster pathogenesis model. Despite the reduced disease, the

$11 \triangle$ PRRA mutant offered robust protection from SARS-CoV-2 rechallenge. Importantly, plaque

12 reduction neutralization tests (PRNT ${ }_{50}$ ) with COVID-19 patient sera and monoclonal antibodies

13 against the receptor-binding domain found a shift, with the mutant virus resulting in consistently

14 reduced $\mathrm{PRNT}_{50}$ titers. Together, these results demonstrate a critical role for the furin cleavage

15 site insertion in SARS-CoV-2 replication and pathogenesis. In addition, these findings illustrate

16 the importance of this insertion in evaluating neutralization and other downstream SARS-CoV-2

17 assays.

\section{Importance}

19 As COVID-19 has impacted the world, understanding how SARS-CoV-2 replicates and causes

20 virulence offers potential pathways to disrupt its disease. By removing the furin cleavage site,

21 we demonstrate the importance of this insertion to SARS-CoV-2 replication and pathogenesis.

22 In addition, the findings with Vero cells indicate the likelihood of cell culture adaptations in virus

23 stocks that can influence reagent generation and interpretation of a wide range of data including

24 neutralization and drug efficacy. Overall, our work highlights the importance of this key motif in

25 SARS-CoV-2 infection and pathogenesis. 


\section{Introduction}

27 The rapid emergence of severe acute respiratory syndrome 2 coronavirus (SARS-CoV-2) at the end of 2019 ushered in a pandemic that has led to over 24 million cases and over 800,000 deaths ${ }^{1,2}$. The novel coronavirus, like its predecessors severe acute respiratory syndrome coronavirus SARS-CoV and Middle East Respiratory Syndrome (MERS)-CoV induces potentially severe respiratory disease including fever, breathing difficulty, bilateral lung infiltration, and in many cases, death ${ }^{3,4}$. While SARS-CoV-2 shares a similar genomic structure and protein homology with SARS-CoV, its ability to spread asymptomatically and cause a range of mild to severe disease distinguishes it from the earlier pandemic $\mathrm{CoV}^{5}$. In exploring the differences, attention has been paid to the spike (S) protein, a key glycoprotein responsible for receptor binding and entry into the cell. Following receptor recognition, the S protein is subsequently cleaved at two sites, S1/S2 and the S2' site to facilitate virus entry into a cell. Initial structural work has indicated that SARS-CoV-2 has greater affinity for the ACE2 receptor than the original SARS-CoV ${ }^{6,7}$. In addition, changes in the $\mathrm{N}$-terminal domain in addition to the receptor-binding domain indicate potential differences in attachment that may drive changes to

41 transmission or virulence ${ }^{8}$. However, the majority of attention has focused on a potentially

42 critical insertion of a furin cleavage site upstream of the $\mathrm{S} 1$ cleavage site in spike ${ }^{9}$. Absent in

43 other group 2B CoVs, the four additional amino acids (PRRA) form the classic RXXR motif

44 cleaved by many serine proteases when added to the conserved R found at the SARS-CoV-2

45 S1 cleavage site (PRRAR) ${ }^{10}$. Notably, furin cleavage sites have been observed in other

46 virulent pathogens like HIV, avian influenza strains ( $\mathrm{H} 5$ and $\mathrm{H} 7)$ as well as Ebola ${ }^{11}$. In fact, furin

47 cleavage sites are found in a number of other CoV family members including MERS-CoV,

48 HKU1-Cov, and OC43-CoV ${ }^{12,13}$; given the range of disease associated with these CoV strains,

49 the furin cleavage site does not necessarily predetermine virulence. However, given its

50 absence in other group 2B CoVs and the major differences in disease compared to SARS-CoV, 
51 a better understanding of the role of the furin cleavage site during SARS-CoV-2 infection is

52 needed.

53 In this manuscript, we utilized a reverse genetic system to generate a SARS-CoV-2

54 mutant that lacked the furin cleavage site insertion ${ }^{14}$. The mutant, $\triangle P R R A$, had augmented

55 replication and improved fitness in Vero E6 cells relative to wild type (WT) SARS-CoV-2. It also

56 had reduced spike processing as compared to the WT virus. In contrast, the $\triangle$ PRRA mutant

57 was attenuated in Calu3 cells, a human respiratory cell line, and had altered S protein

58 processing as compared to Vero cells. In vivo, the $\triangle \mathrm{PRRA}$ mutant had attenuated disease in

59 hamsters despite robust, and sometimes augmented viral replication. Importantly, prior

60 infection with the $\triangle \mathrm{PRRA}$ mutant protected hamsters from subsequent rechallenge with WT

61 SARS-CoV-2. Finally, neutralization assays that used the $\triangle P R R A$ mutant had lower PRNT 50

62 values with both COVID-19 patient sera and monoclonal antibodies against the receptor-binding

63 domain (RBD). Together, the results indicate a critical role for the furin cleavage site in SARS-

64 CoV-2 infection and potential complications in interpreting research related to this virus

65 infection. 


\section{Results}

68 The SARS-CoV-2 spike protein is $>75 \%$ conserved in amino acid sequence across the group

$692 \mathrm{~B} \mathrm{CoV}$ family with the majority of the differences occurring in the $\mathrm{N}$-terminal domain and

70 receptor-binding domain in the S1 portion (S. Fig. 1A). The insertion of a furin cleavage site

71 (PRRA) upstream of the S1 cleavage site distinguishes SARS-CoV-2 from other group 2B CoV

72 sequences including SARS-CoV and RATG13, the closest bat-derived CoV sequence (S. Fig.

73 1B). To evaluate the impact of the furin cleavage site insertion, we generated a mutant virus

74 lacking the PRRA motif using our SARS-CoV-2 reverse genetic system (Fig. 1A) ${ }^{14}$. Based on

75 the SARS-CoV S protein structure, the insertion occurs in an exterior loop of the SARS-CoV-2

76 spike below the spike globular head and away from the receptor binding domain (Fig. 1B) ${ }^{15}$.

77 Using homology modeling based on the SARS-CoV S protein structure, we found the PRRA

78 insertion in an extended loop (cyan). Deletion of PRRA insertion is predicted to shorten the

79 loop, but importantly, not disrupt the overall structure of the spike protein. Following

80 electroporation, we were able to recover the SARS-CoV-2 $\triangle \mathrm{PRRA}$ mutant with stock virus titer

81 roughly equivalent to the wild-type (WT) virus. Surprisingly, the SARS-CoV-2 $\triangle P R R A$ mutant

82 virus produced a larger plaque size on Vero E6 cells than WT virus, suggesting potential

83 changes in viral replication and spread in the absence of the furin cleavage site insertion (S.

84 Fig. 1C).

85 Distinct replication kinetics and spike cleavage for the $\triangle$ PRRA mutant in Vero E6 cells.

86 To evaluate viral replication, we infected Vero E6 cells with the WT and $\triangle$ PRRA SARS-CoV-2.

87 Previous work has found robust replication of SARS-CoV-2 in Vero E6 cell and these cells are

88 often used for propagation, including for inactivated vaccine production ${ }^{16}$. Following low MOI

89 (0.01 plaque forming units (PFU)/cell) infection, both WT and $\triangle$ PRRA SARS-CoV-2 mutant

90 replicated to similar end-point titers (Fig. 1C). However, the $\triangle P R R A$ had a 25-fold increase in

91 viral titer at 24 hours post infection (HPI) relative to WT. The increased replication was 
92

93

94

accompanied by more cytopathic effect (CPE) at $24 \mathrm{HPI}$; by $48 \mathrm{HPI}$, both mutant and WT levels had nearly $100 \%$ CPE. Together, the results suggested the loss of the furin cleavage site augmented replication in Vero E6 cells.

We next evaluated spike processing of the $\triangle \mathrm{PRRA}$ mutant relative to WT SARS-CoV-2 as well as the original SARS-CoV. Vero E6 cells were infected at an MOI of $\sim 0.1$ for $24 \mathrm{~h}$ and purified virions were isolated from supernatants using ultracentrifugation and a sucrose cushion. The pelleted virus was subsequently examined for spike and nucleocapsid ( $\mathrm{N}$ ) protein levels by western blotting. Following SARS-CoV infection, the majority of the spike protein was observed in its full-length form (98.6\%) (Fig. 1D, S. Fig. 2A), consistent with the absence of processing. In contrast, the WT SARS-CoV-2 virions had a significant reduction in full-length spike protein (40.4\%). Instead, the most abundant form of the spike protein was the S1/S2 cleavage product (59.6\%). Finally, the $\triangle$ PRRA mutant spike protein had mostly full-length spike $(85.5 \%)$, similar to SARS-CoV, with only minimal processing to the S1/S2 cleavage form (14.5\%). Given similar levels of viral $\mathrm{N}$ protein, these results illustrate the differences in processing between SARSCoV and SARS-CoV-2. In addition, the data show that processing of the SARS-CoV-2 spike is driven primarily by the furin cleavage site following infection of Vero E6 cells.

Given the replication advantage noted at $24 \mathrm{HPI}$ (Fig. 1D), we next evaluated the fitness of the $\triangle P R R A$ mutant relative to WT SARS-CoV-2 in a competition assay. Using plaqueforming units to determine the input, we mixed the WT and mutant viruses at different ratios in Vero E6 cells, and used a RT-PCR approach to evaluate their overall fitness after 24 hours (Fig. 1E, S. Fig 2B-C). At a 50:50 input ratio, the $\triangle$ PRRA mutant quickly outcompeted WT becoming nearly $90 \%$ of the viral population based on both RT-PCR distinguishing the two viruses. Similarly, a $90: 10$ WT to mutant input ratio resulted in $\sim 65 \%$ of the viral sequences corresponding with the mutant virus, illustrating the advantage of the furin site deletion after only 24 HPI. The inverse 10:90 WT to mutant input ratio produced $<3 \%$ of the WT virus and solidified 
117 the major advantage of the $\triangle$ PRRA mutant in Vero E6 cells. We further confirmed these data

118 using deep sequencing analysis (S. Fig. 2D). Together, the results indicate that deletion of the

119 furin cleavage site provides a fitness advantage in Vero E6 cells and a potentially potent cell

120 culture adaptation.

121 Attenuation of SARS-CoV-2 $\triangle$ PRRA in Calu3 respiratory cells. Having established 122 augmented replication, altered spike processing, and enhanced fitness in Vero E6 cells, we next 123 evaluated the $\triangle$ PRRA mutant in a more relevant cell type. Previously, Calu3 2B4 cells, a 124 human lung adenocarcinoma cell line, had been sorted for ACE2 expression and used to study 125 influenza and coronaviruses ${ }^{17,18}$. In this study, we infected Calu3 2B4 with WT and $\triangle$ PRRA 126 SARS-CoV-2 at MOI 0.01. Following infection, we found robust replication of WT SARS-CoV-2 127 peaking $72 \mathrm{HPI}$. In contrast, the $\triangle \mathrm{PRRA}$ mutant virus was attenuated relative to WT beginning $12848 \mathrm{HPI}$ (Fig. 1F). At both 48 and $72 \mathrm{HPI}$, the $\triangle \mathrm{PRRA}$ mutant had 1-log reduction in viral titer, 129 contrasting the improved replication observed in Vero E6 cells. These data indicate that the loss 130 of the furin cleavage site impairs replication in Calu3 cells and suggests SARS-CoV-2 requires 131 the PRRA motif for efficient replication in these respiratory cells.

We subsequently repeated examination of the spike processing on virions from Calu3 133 2B4 cells for the $\triangle$ PRRA mutant. Similar to studies with Vero E6 cells, we infected Calu3 2B4 134 cells with SARS-CoV, SARS-CoV-2 WT, and $\triangle$ PRRA mutants at MOI 0.1. Given the reduced 135 viral yields in Calu3 2B4 (Fig. 1F), we allowed replication to occur until 48 HPI before capturing 136 supernatants and recovering purified virions. Consistent with findings in Vero E6 cells, the 137 western blot of SARS-CoV virions showed the majority of spike protein was retained in the full138 length form (Fig. 1G, S. Fig. 2E). In contrast, the WT SARS-CoV-2 showed the majority of its 139 spike protein had been cleaved to the S1/S2 form. Comparing to Vero E6 cells, spike 140 processing to the $\mathrm{S} 1 / \mathrm{S} 2$ form was more robust in Calu3 showing a $\sim 87.3 \%$ at $\mathrm{S} 1 / \mathrm{S} 2$ as 141 compared to $59.6 \%$ in Vero E6 cells. Surprisingly, the $\triangle$ PRRA mutant also showed a significant 
142 increase in the S1/S2 cleavage product relative to its Vero spike blots. While the $\triangle \mathrm{PRRA}$ has

143 reduced overall infection, a clear band was visible at the $\mathrm{S} 1 / \mathrm{S} 2$ cleavage site and represents

144 more than 2 times as much S1/S2 cleavage product (33.1\% vs $14.5 \%)$ compared to Vero E6

145 results. While more full-length spike is observed in the WT virus infection, the Calu3 results

146 indicate that even without the furin cleavage site, there is significant processing of the SARS-

147 CoV-2 spike. The results suggest that while the furin cleavage site is key in SARS-CoV-2 spike

148 processing, other factors outside the PRRA insertion play a role in the efficient cleavage of the

149 SARS-CoV-2 spike in a cell type-dependent manner.

150 In vivo attenuation of $\triangle$ PRRA mutant. Having established contrasting results with in vitro

151 studies, we next sought to evaluate the SARS-CoV-2 $\triangle$ PRRA mutant in an in vivo model. Early

152 attempts found mouse models non-viable for SARS-CoV-2 infection ${ }^{19}$; therefore we shifted to

153 the hamster model which shows modest disease following infection with SARS-CoV-2 infection

15420 . Four male hamsters were challenged with $10^{5}$ PFU of either WT SARS-CoV-2 or $\triangle P R R A$

155 mutant (Fig. 2A). The animals were subsequently monitored for 28 days with periodic measures

156 of their body weight and disease signs. In addition, nasal washes and oral swabs were taken at

157 day 2-7, 14, 21, and 28 days post infection (DPI). Following infection with WT SARS-CoV-2,

158 hamsters steadily lost weight starting at day 2 and continuing through day 8 with peak weight

159 loss nearing 15\% (Fig. 2B, S. Fig 3A). These WT-infected hamsters also had disease scores

160 that peaked between days 8 and 10, when animals showed signs including ruffled fur, hunched

161 posture, and reduced activity requiring additional monitoring (Fig. 2C, S. Fig. 3B). Despite this

162 severe disease, the WT-infected hamsters subsequently recovered and regained their starting

163 weight by day 15 (S. Fig. 3A). In contrast, hamsters infected with SARS-CoV-2 $\triangle$ PRRA

164 showed minimal weight loss over the course of infection (Fig. 3B, S. Fig. 3A). Over the first

165 four days of infection, the $\triangle P R R A$ infected hamsters showed $2-3 \%$ weight loss, but remained

166 close to their starting weight through day 10. In addition, the $\triangle P R R A$ mutant-infected hamsters 
167 had no change in disease score over the course of infection, distinguishing it from symptomatic

168 disease observed following WT SARS-CoV-2 infection. The hamsters in both groups eventually 169 gained a significant amount of weight after day 10 over the remainder of the 28-day time course 170 (S. Fig. 3A).

171 Despite attenuated disease, the viral titers revealed augmented replication of the $172 \Delta$ PRRA mutant relative to WT SARS-CoV-2. Examining nasal washes, both WT and $\triangle \mathrm{PRRA}$ 173 infected hamsters had similar viral titers 2 DPI (Fig. 2D). However, augmented $\triangle P R R A$ 174 replication was observed at both days 3 and 4 relative to the WT SARS-CoV-2. In addition, the 175 WT virus was cleared from the nasal washes a day earlier than the $\triangle$ PRRA mutant, although no 176 plaque forming units were detected after day 7 in either of the hamster groups. Evaluating oral 177 swabs for viral RNA, a similar pattern was observed with augmented viral RNA at days 3 and 4 178 in the $\triangle$ PRRA mutant relative to WT (Fig. 2E). Notably, the viral RNA in the swabs stayed 179 positive though day 7 with augmented WT viral RNA yield observed at the latest time point 180 relative to $\triangle \mathrm{PRRA}$. Together, the results suggest that despite the attenuation in disease, the $181 \quad \triangle$ PRRA mutant is capable of robust replication in the oral and nasal cavity of hamsters following 182 infection.

183 Infection with $\triangle$ PRRA mutant protects from SARS-CoV-2 rechallenge. We next evaluated if 184 infection with $\triangle P R R A$ offered protection from further SARS-CoV-2 infection. Hamsters 185 previously infected with either WT SARS-CoV-2 or the $\triangle$ PRRA mutant were rechallenged with $18610^{5}$ PFU of WT SARS-CoV-2 28 days post initial infection (Fig. 2F). The rechallenged hamsters 187 were monitored for disease and weight loss over a 21-day time course. Contrasting initial 188 challenge, both WT and $\triangle \mathrm{PRRA}$ infected hamsters were protected from weight loss following 189 rechallenge (Fig. 2G and S. Fig. 3B). Hamsters infected with WT SARS-CoV-2 initially had no 190 weight loss over the course of infection; however, mild disease (ruffled fur) was observed in a 191 subset of animals (Fig. 2H). In contrast, rechallenge of $\triangle$ PRRA mutant-infected hamsters 
192 produced neither weight loss nor any evidence of disease. Nasal wash titers and viral RNA from

193 oral swabs showed viral replication in both WT- and $\triangle$ PRRA-infected animals at day 2 and 3;

194 however, the overall viral loads were significantly reduced compared to initial challenge (Fig. 2l

195 \& J). In addition, plaque forming virus appeared to be cleared around day 4 following

196 rechallenge with low viral RNA loads found in oral swabs at corresponding time points. The

197 results indicate that infection with the $\triangle \mathrm{PRRA}$ mutant protects hamsters from disease upon

198 rechallenge, but does not provide sterilizing immunity.

199 Loss of furin cleavage site alters COVID19 serum neutralization. We next sought to

200 evaluate the impact of the furin cleavage site deletion on virus neutralization by COVID-19

201 patient sera and monoclonal antibodies (mAB) against the SARS-CoV-2 receptor binding

202 domain (RBD). To quantitate neutralization, we generated a $\triangle P R R A$ mutant containing the

203 mNeonGreen ( $\mathrm{mNG}$ ) reporter in open reading frame 7 and subsequently compared

204 neutralization results to the WT SARS-CoV-2 mNG reporter assay as previously described ${ }^{21}$

205 (Fig. 3A). Examining seventeen COVID-19 human sera samples, we found nearly uniform

206 reduction in PRNT $_{50}$ values against the $\triangle \mathrm{PRRA}$ mutant as compared to the WT control virus

207 (Fig. 3B). The lower $\mathrm{PRNT}_{50}$ values were observed in sera whether the samples were from low,

208 intermediate, or high neutralizing COVID19 patients (Fig. 3C-E) and averaged a 2.3-fold

209 reduction across the 17 human sera. The consistency in the reduction suggested several

210 possibilities: One is that virions themselves are altered in the conformation of the spike on the

211 surface. In this situation, the processing or loss of spikes may permit access to more cryptic

212 sites on the spike S2 or other regions allowing the WT virus to be more easily neutralized by

213 non-receptor binding domain antibodies. A second possibility is that the loss of the furin

214 cleavage site leaves more intact spike molecules on the virion surface requiring more antibodies

215 to neutralize the $\triangle \mathrm{PRRA}$ mutant than WT SARS-CoV-2. To explore this question, we examined

216 the $\triangle \mathrm{PRRA}$ mutant neutralization in the presence of three monoclonal antibodies $(\mathrm{mAB})$ that 
217 target the SARS-CoV-2 receptor binding domains (RBD) (Fig. 3F-H). Each mAB targets a 218 different site in the RBD, but each had similar reduction in the mAB serum neutralization levels

219 between WT and $\triangle \mathrm{PRRA}$. The need for more mAB or polyclonal COVID patient sera suggest

220 that $\triangle$ PRRA has more spike proteins that must be neutralized. Together, the results highlight

221 significant differences in the neutralization between the WT and $\triangle$ PRRA SARS-CoV-2 mutants. 


\section{Discussion}

224 The loss of the furin cleavage site in the SARS-CoV-2 spike has a major impact on infection and 225 pathogenesis. Using a reverse genetic system for the SARS-CoV-2 WA1 isolate, we generated 226 a mutant virus that deleted the four amino acid insertion ( $\triangle \mathrm{PRRA}$ ). The loss of the furin 227 cleavage site resulted in reduced infection in Calu3 respiratory cells and ablated disease in the 228 hamster pathogenesis model of SARS-CoV-2. Despite attenuated disease on initial infection, 229 the $\triangle$ PRRA infected hamsters were protected from subsequent challenge with WT SARS-CoV-2 230 indicating induction of robust immunity. Together, the results highlight the importance of the 231 furin cleavage site insertion to SARS-CoV-2 infection and pathogenesis.

Notably, despite attenuated disease in vivo, the $\triangle \mathrm{PRRA}$ mutant had advantages over WT SARS-CoV-2 and may complicate research studies. The $\triangle$ PRRA mutant has a fitness

234 advantage over the WT strain and dominated in vitro competition assays in Vero E6 compared 235 to WT virus. Importantly, the furin site deletion has been reported in SARS-CoV-2 preparations

$236{ }^{22}$ and given its fitness advantage in Vero E6 cells, can easily become the dominant tissue 237 culture adaption in virus preparations. Coupled with in vitro and in vivo attenuation, efforts must 238 be made to verify and evaluate stocks prior to critical studies. This also has implications for 239 manufacturing inactivated COVID19 vaccine on Vero cells ${ }^{23}$. Similarly, the shift in antibody 240 neutralization values of the $\triangle P R R A$ virus indicates the possibility of inaccurate results if this 241 mutation appears and distinguishes results from pseudotyped particles with and without this 242 furin mutations ${ }^{24}$. Fortunately, the $\triangle P R R A$ mutation will under represent the level of SARS-

243 CoV-2 neutralization rather than overstating protection level; however, with potential vaccine 244 and therapeutics decisions resting on these PRNT $_{50}$ values, accuracy must be paramount. 245 Together, the data highlight the importance of recognizing the mutation for future SARS-CoV-2 246 experimental analysis. 
Biologically, the loss of the furin site shifts the processing of the spike in a cell type dependent manner. In Vero E6 cells, $\triangle$ PRRA significantly reduces cleavage to the S1/S2 form on the virion and the spike protein remains in the full-length conformation mirroring the results observed for SARS-CoV. In contrast, WT SARS-CoV-2 processes nearly $60 \%$ of its spike to S1/S2 indicating that most spike on the virion surface have been cleaved. Notably, the spike processing is distinct in Calu3 2B4 respiratory cells. While SARS-CoV virions remain uncleaved with little S1/S2 cleavage product, WT SARS-CoV-2 has increased processing from full-length

254 to the S1/S2 cleavage product than what was observed in Vero E6 cells. Surprisingly, $\triangle \mathrm{PRRA}$ 255 in Calu3 cells also had a shift toward the S1/S2 fragment which is absent in Vero E6 cells. The 256 results indicate that while the majority of the spike cleavage in SARS-CoV-2 is mediated by the 257 furin cleavage site, there is more spike processing in SARS-CoV-2 even in its absence. With 258 known serine protease differences between Vero E6 and Calu3 2B4 cells ${ }^{25}$, the results suggest 259 that spike processing varies based on cell type and may contribute to altered infection and 260 pathogenesis in vivo.

In hamsters, the loss of the furin site attenuates SARS-CoV-2 induced disease, but does not ablate $\triangle \mathrm{PRRA}$ virus replication. Following challenge, hamsters infected with the $\triangle \mathrm{PRRA}$ had

263 minimal change in weight loss over the first 10 DPI. In contrast, WT SARS-CoV-2 infected 264 hamsters lost $\sim 15 \%$ of their body weight and showed signs of disease (hunching, diminished 265 movement, ruffled fur). While WT infected hamsters recovered, the absence of disease in the $266 \triangle \mathrm{PRRA}$ infected hamsters indicates a key role for the furin cleavage site in virulence. 267 Surprisingly, the $\triangle \mathrm{PRRA}$ mutant was not attenuated in virus replication. At 3 and $4 \mathrm{DPI}$, the $268 \triangle \mathrm{PRRA}$ had augmented titers as compared to control in nasal washes and increased viral RNA 269 in the oral swabs. Similarly, the virus cleared one day later than WT SARS-CoV-2 during 270 primary challenge. The results suggest that the reduced disease observed following $\triangle P R R A$ 271 challenge was not a result of attenuated replication in these tissues. 
273 protected from further WT SARS-CoV-2 infection. After 28 days, $\triangle$ PRRA and WT infected

274 hamsters were rechallenged with WT SARS-CoV-2 and were protected from weight loss. While

275 mild disease was observed in one of the WT SARS-coV-2 infected hamsters, the $\triangle \mathrm{PRRA}$

276 infected hamsters showed no evidence of disease. However, low viral loads were observed in

277 both the nasal washes and oral swabs from both groups, suggesting that the hamsters could

278 foster a low level of infection after rechallenge. Yet, the virus was rapidly cleared and failed to

279 induce disease in both groups, suggesting that adequate protection had been induced.

280 Together, the results suggest that $\triangle \mathrm{PRRA}$ mutant, despite attenuated disease, induces

281 sufficient immunity to protect hamsters from further SARS-CoV-2 infection.

Overall, the data presented in this manuscript illustrate the critical role the furin cleavage

283 site insertion in the spike protein plays in SARS-CoV-2 infection and pathogenesis. In its

284 absence, the mutant $\triangle P R R A$ virus is attenuated in its ability to replicate in certain cell types and

285 to cause disease in vivo. However, the results are complicated by augmented replication and

286 fitness in Vero cells. Similarly, altered antibody neutralization profiles indicate a critical need to

287 survey this mutation in analysis of SARS-CoV-2 treatments and vaccines moving forward.

288 Together, the work highlights the critical nature of the furin cleavage site in understanding

289 SARS-CoV-2 infection and pathogenesis. 


\section{Methods}

292 Viruses and cells. The recombinant wild-type and mutant SARS-CoV-2 are based on the 293 sequence of USA-WA1/2020 isolate provided by the World Reference Center for Emerging 294 Viruses and Arboviruses (WRCEVA) and was originally obtained from the USA Centers of 295 Disease Control as described ${ }^{16}$. Wild-type and mutant SARS-CoV-2 as well as recombinant 296 mouse-adapted recombinant SARS-CoV ${ }^{26}$ were titrated and propagated on Vero E6 cells, 297 grown in DMEM with 5\% fetal bovine serum and 1\% antibiotic/antimytotic (Gibco). Calu3 2B4 298 cells were grown in DMEM with 10\% defined fetal bovine serum, 1\% sodium pyruvate (Gibco), 299 and 1\% antibiotic/antimitotic (Gibco). Standard plaque assays were used for SARS-CoV and 300 SARS-CoV-2 ${ }^{27,28}$. All experiments involving infectious virus were conducted at the University of 301 Texas Medical Branch (Galveston, TX) or Emory University (Atlanta, Georgia) in approved 302 biosafety level 3 (BSL) laboratories with routine medical monitoring of staff.

303 Construction of $\triangle$ PRRA Mutant Viruses. Both wild-type and mutant viruses were derived

304 from the SARS-CoV-2 USA-WA1/2020 infectious clone as previously described ${ }^{14}$. For $\triangle P R R A$ 305 mutant construction, the mutation was introduced into a subclone puc57-CoV2-F6 by using 306 overlap PCR with primers $\triangle$ PRRA-F (5ロ-GACTAATTCTCGTAGTGTAGCTAGTCAATCCATC-

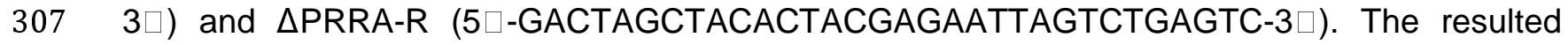
308 plasmid was validated by restriction enzyme digestion and Sanger sequencing. Thereafter, plasmids containing wild-type and mutant SARS-CoV-2 genome fragments were amplified and restricted. The SARS-CoV-2 genome fragments were purified and ligated in vitro to assemble

311 the full-length cDNA according to the procedures described previously ${ }^{14}$. In vitro transcription

312 reactions were then preformed to synthesize full-length genomic RNA. To recover the viruses,

313 the RNA transcripts were electroporated into Vero E6 cells. The media from electroporated cells 314 were harvested at 40-hour post-infection and served as seed stocks for subsequent 315 experiments. Viral mutants were confirmed by sequence analysis prior to use. Synthetic 
construction of SARS-CoV-2 $\triangle$ PRRA mutant was approved by the University of Texas Medical Branch Institutional Biosafety Committee.

In Vitro Infection. Viral replication in Vero E6 and Calu3 2B4 cells were performed as

319 previously described ${ }^{29,30}$. Briefly, cells were washed with PBS and inoculated with SARS-CoV or SARS-CoV-2 at a multiplicity of infection (MOI) 0.01 for 60 minutes at $37{ }^{\circ} \mathrm{C}$. Following

321 inoculation, cells were washed, and fresh media was added to signify time 0 . Three or more

322 biological replicates were harvested at each described time. No blinding was used in any

323 sample collections, nor were samples randomized.

Virion Purification and Western Blot. Vero E6 or Calu3-2B4 cells were infected with WT or PRRA mutant viruses at an MOI of 0.01 . At $24 / 48 \mathrm{HPI}$, the culture media were collected and clarified by low speed spin. Virus particles in the media were subsequently pelleted by

327 ultracentrifugation through a $20 \%$ sucrose cushion at 26,000 rpm for $3 \mathrm{~h}$ by using a Beckman

328 SW28 rotor. For western blot analysis, protein lysates were prepared from the pellets using $2 \mathrm{X}$

329 Laemmli Sample buffer (Cat\# 161-073, BioRad, Hersules,Ca). Relative viral protein levels were 330 then determined by SDS-Page followed by western blot analysis as previously described ${ }^{16,31}$. 331 Briefly, sucrose purified SARS-CoV-1, SARS-CoV-2, and SARS-CoV-2 $\triangle$ PRRA inactivated by 332 boiling in Laemelli Buffer. Samples were loaded in equal volumes into $4-20 \%$ Mini-PROTEAN 333 TGX Gels (Biorad\# 4561093) and electrophoresed by SDS-Page. Protein was then transferred 334 to polyvinylidene difluoride (PVDF) membranes. Membranes were then blotted with SARS-CoV 335 Spike (S) specific antibodies (Novus Biologicals \#NB100-56576), followed by probing with 336 horseradish peroxidase (HRP)-conjugated anti-rabbit antibody (Cell Signaling Technology 337 \#7074S) as a secondary. Blots were then stripped and re-probed with SARS-CoV Nucleocapsid 338 (N) specific antibodies (provided as a kind gift from Dr. Shinji Makino) and the HRP-conjugated 339 anti-rabbit secondary. In both cases, signal was developed by treating membranes with Clarity 
Western ECL substrate (Bio-Rad \#1705060) imaging on a ChemiDoc MP System (Bio-Rad \#12003154). Densitometry was performed using ImageLab 6.0.1 (Bio-Rad \#12012931).

342 Competition Assay and Real-Time PCR. For competition assays, ratios (50:50, 90:10, 10:90

343 WT/ $\triangle \mathrm{PRRA}$ ) were determined by plaque forming units derived from viral stocks. Vero cells

344 were infected at $\mathrm{MOI} 0.1(\mathrm{WTn}+\triangle \mathrm{PRRA})$ as described above. RNA from cell lysates were 345 collected using Trizol reagent (Invitrogen). RNA was then extracted from Triazol using the

346 Direct-zol RNA Miniprep Plus kit (Zymo Research \#R2072) per the manufacturer's instruction.

347 Extracted RNA was then converted to cDNA with the iScript cDNA Synthesis kit (BioRad 348 \#1708891). Quantitative real time PCR (qRT-PCR) was performed with the Luna Universal 349 qPCR Master Mix (New England Biolabs \#M3003) on a CFX Connect instrument (BioRad 350 \#1855200). For differentiation between wild type SARS-CoV-2 and SARS-CoV-2 $\triangle P R R A$ 351 genomes in competition experiments, Primer 1 (Forward - AAT GTT TTT CAA ACA CGT GCA 352 G and Primer 2 (Reverse - TAC ACT ACG TGC CCG CCG AGG) were used to detect wild type 353 genomes only. For detecting total genomes, Primer 1 and Primer 3 (Reverse - GAA TTT TCT 354 GCA CCA AGT GAC A) were used. 8-point standard curves ( $1 \times 10^{1}$ to $1 \times 10^{8}$ copies/ $\left.\mu \mathrm{L}\right)$ were 355 utilized to quantify the signal. A primer annealing temperature of $63^{\circ} \mathrm{C}$ was used for all assays.

356 For detection of viral RNA the nasal washes and oral swabs of SARS-CoV-2 and SARS-CoV-2 $357 \quad \triangle P R R A$ infected hamsters, RNA extraction, cDNA synthesis, and qRT-PCR were performed as 358 described above. For qRT-PCR, Primer 1 and Primer 3 were utilized for all hamster samples.

359 Deep Sequencing Analysis. RNA libraries were prepared with 300ng of RNA using the Click-

360 Seq protocol as previously described ${ }^{32}$ using tiled primers cognate to the SARS-COV-2 361 genome (accession number NC_045512.2) and the TruSeq i7 LT adapter series and i5 362 hexamer adaptors containing a 12N unique molecular identifier (UMI). Libraries were 363 sequenced on the Illumina MiSeq platform with MiSeq Reagent Kit v2. Raw data was de-

364 multiplexed using TruSeq indexes using the MiSeq Reporter Software. Fastp v0.12. ${ }^{33}$ was used 
365 to trim adapter sequences and low-quality reads $(q<25)$, remove reads less than $40 \mathrm{nts}$ in

366 length, and copy UMI sequences onto the read name. Reads were aligned with bowtie using the

367 -best parameter and allowing for up to two mismatches. The alignment index was generated

368 from a single fasta file, which contained two 600nt reference sequences spanning the PRRA

369 locus (23603-23616) of the wildtype (accession number NC_045512.2) and $\Delta$ PRRA genomes.

370 The alignments were sorted and indexed using Samtools v1.9 ${ }^{34}$, PCR duplicates were removed

371 using umi_tools ${ }^{35}$. Coverage at each position was determined with the genomecov function in

372 bedtools v2.25.0 ${ }^{36}$.

373 Plaque reduction neutralization titer assay. Neutralization assays were preformed using

374 mNeonGreen SARS-CoV-2 reporter neutralization assay as previously described ${ }^{21}$. Briefly,

375 Vero E6 cells were plated black $\mu$ CLEAR flat-bottom 96-well plate (Greiner Bio-one ${ }^{\mathrm{TM}}$ ). On

376 following day, sera or monoclonal antibodies were serially diluted from 1/20 with nine 2-fold

377 dilutions to the final dilution of $1 / 5120$ and incubated with mNeonGreen SARS-CoV-2 or $\triangle P R R A$

378 expressing $\mathrm{mNeonGreen}$ at $37^{\circ} \mathrm{C}$ for $1 \mathrm{~h}$. The virus-serum mixture was transferred to the Vero

379 E6 cell plate with the final multiplicity of infection (MOI) of 0.5 . After 20 hours, Hoechst 33342

380 Solution (400-fold diluted in Hank's Balanced Salt Solution; Gibco) was added to stain cell

381 nucleus, sealed with Breath-Easy sealing membrane (Diversified Biotech), incubated at $37^{\circ} \mathrm{C}$ for

$38220 \mathrm{~min}$, and quantified for $\mathrm{mNeonGreen}$ fluorescence on Cytation ${ }^{\mathrm{TM}} 7$ (BioTek). The raw images

383 ( $2 \times 2$ montage) were acquired using $4 \times$ objective, processed, and stitched using the default

384 setting. The total cells (indicated by nucleus staining) and mNeonGreen-positive cells were

385 quantified for each well. Infection rates were determined by dividing the mNeonGreen-positive

386 cell number to total cell number. Relative infection rates were obtained by normalizing the

387 infection rates of serum-treated groups to those of non-serum-treated controls. The curves of

388 the relative infection rates versus the serum dilutions $\left(\log _{10}\right.$ values) were plotted using Prism 8

389 (GraphPad). A nonlinear regression method was used to determine the dilution fold that

390 neutralized $50 \%$ of mNeonGreen fluorescence (NT50). Each serum was tested in duplicates. 

constructed from a set of representative group 2B coronaviruses by using alignment data paired with neighbor-joining phylogenetic trees built in Geneious (v.9.1.5) using the spike amino acid sequences derived the following accession numbers: QHU79204 (SARS-CoV-2 WA1), QHR63300.2 (RATG13), QND76034.1 (HKU3), AGZ48828.1 (WIV1), AGZ48806 (RsSHC014), ALK02457 (WIV16), and AYV99817.1(SARS-CoV Urbani). Sequence identity was visualized using EvolView (http://evolgenius.info/) and utilized SARS-CoV Co-V-2 WA1 as the reference sequence. Tree shows the degree of genetic similarity of SARS-CoV-2 and SARS-CoV across a selected group 2B coronaviruses. Structural models were generated using SWISS-Model ${ }^{37,38}$ to generate homology models for SARS-CoV-2 spike with and without the furin cleavage site insertion based on the SARS-CoV-1 trimer structure (PDB 6ACD). Homology models were visualized and manipulated in MacPyMol (version 1.3).

403 Animals and ethics statements. Male Syrian hamsters (7-8 weeks old, 86-127 $\square$ g) were 404 purchased from Envigo. All procedures were conducted under an animal protocol approved by 405 the UTMB Institutional Animal Care and Use Committee and complied with USDA guidelines in an AAALAC-accredited lab. Work with infectious SARS-CoV-2 in hamsters was performed in 407 the Galveston National Laboratory BSL-4 laboratory. Animals were housed in microisolator 408 caging equipped with HEPA filters in the BSL-4 laboratories.

409 Hamster Infection studies. Hamsters were challenged with $10^{5}$ PFU of WT-SARS-CoV-2 or 410 SARS-CoV-2 $\triangle$ PRRA by intranasal inoculation (i.n.). Hamsters were observed daily for the 411 development of clinical disease and body weights were taken every day for the first 10 days of 412 the study, then every third day. For each manipulation (viral infection, retro-orbital bleeds, nasal 413 wash, or oral swab), animals were anesthetized with isoflurane (Piramal, Bethlehem, PA).

414 Statistical analysis. All statistical comparisons in this manuscript involved the comparison 415 between 2 groups, SARS-CoV or SARS-CoV-2 infected groups under equivalent conditions. 
416 Thus, significant differences in viral titer were determined by the unpaired two-tailed students T-

417 Test.

418 Data Availability. The raw data that support the findings of this study are available from the

419 corresponding author upon reasonable request.

420 Acknowledgements. Research was supported by grants from NIA and NIAID of the NIH to 421 (Al153602 and AG049042 to VDM Al142759, Al134907, Al145617, and UL1TR001439 to P-

422 YS; R01Al123449 to AF and BL; R24Al120942 (WRCEVA) to SCW). Research was also

423 supported by STARs Award provided by the University of Texas System to VDM and trainee

424 funding provided by the McLaughlin Fellowship Fund at UTMB. P-YS was also supported by

425 CDC grant for the Western Gulf Center of Excellence for Vector-Borne Diseases, and awards

426 from the Sealy \& Smith Foundation, Kleberg Foundation, John S. Dunn Foundation, Amon G.

427 Carter Foundation, Gilson Longenbaugh Foundation, and Summerfield Robert Foundation.

\section{Author Contributions}

429 Conceptualization, XX, BAJ, ALR, MS, ANF, P-YS, and VDM; Methodology, BAJ, XX, BK, KGL, 430 DS, ALR, ANF, P-YS and VDM.; Investigation, BAJ, XX, BK, KGL, AM, JZ, XZ, TJ, JKS, LZ, CS, $431 \mathrm{MV}, \mathrm{AV}, \mathrm{DS}, \mathrm{NB}, \mathrm{JAP}, \mathrm{ALR}, \mathrm{KD}$, and VDM.; Resources, KSP, SCW, MSS, PR, ZK, ZA, P-YS, 432 ANF, VDM; Data Curation, BAJ, XX, BK, KGL, AV, DS, ALR, MSS, KD, P-YS, ANF, VDM.; 433 Writing-Original Draft, VDM; Writing-Review \& Editing, BAJ, XX, BL, PA, MSS, KD, ZK, ZA, P434 YS, ANF, VDM.; Visualization, XX, BAJ, BK, KGL, NB, ANF, VDM; Supervision, PA, SCW, 435 MSS, P-YS, ANF, VDM.; Funding Acquisition, PA, SCW, PY-S, ANF, VDM.

\section{Competing interests}

437 X.X., V.D.M., and P.-Y.S. have filed a patent on the reverse genetic system and reporter SARS438 CoV-2. Other authors declare no competing interests. 
441 1. Gralinski, L.E. \& Menachery, V.D. Return of the Coronavirus: 2019-nCoV. Viruses

442 12(2020).

443 2. Dong, E., Du, H. \& Gardner, L. An interactive web-based dashboard to track COVID-19

444 in real time. Lancet Infect Dis 20, 533-534 (2020).

445 3. Zhu, N., et al. A Novel Coronavirus from Patients with Pneumonia in China, 2019. N

446 Engl J Med 382, 727-733 (2020).

447 4. Huang, C., et al. Clinical features of patients infected with 2019 novel coronavirus in

448 Wuhan, China. Lancet 395, 497-506 (2020).

449 5. Gao, Z., et al. A Systematic Review of Asymptomatic Infections with COVID-19. J

$450 \quad$ Microbiol Immunol Infect (2020).

4516 . Shang, J., et al. Structural basis of receptor recognition by SARS-CoV-2. Nature 581, 452 221-224 (2020).

4537 7. Yuan, M., et al. A highly conserved cryptic epitope in the receptor binding domains of 454 SARS-CoV-2 and SARS-CoV. Science 368, 630-633 (2020).

455 8. Lu, G., Wang, Q. \& Gao, G.F. Bat-to-human: spike features determining 'host jump' of 456 coronaviruses SARS-CoV, MERS-CoV, and beyond. Trends in microbiology 23, 468-478

457 (2015).

$458 \quad 9 . \quad$ Coutard, B., et al. The spike glycoprotein of the new coronavirus 2019-nCoV contains a furin-like cleavage site absent in CoV of the same clade. Antiviral Res 176, 104742 (2020). 10. Seidah, N.G. \& Chretien, M. Proprotein and prohormone convertases: a family of subtilases generating diverse bioactive polypeptides. Brain Res 848, 45-62 (1999).

11. Braun, E. \& Sauter, D. Furin-mediated protein processing in infectious diseases and cancer. Clin Transl Immunology 8, e1073 (2019).

12. de Haan, C.A., et al. Cleavage of group 1 coronavirus spike proteins: how furin cleavage is traded off against heparan sulfate binding upon cell culture adaptation. Journal of virology $\mathbf{8 2}$, 6078-6083 (2008).

13. Kirchdoerfer, R.N., et al. Pre-fusion structure of a human coronavirus spike protein. Nature 531, 118-121 (2016).

14. Xie, X., et al. An Infectious cDNA Clone of SARS-CoV-2. Cell host \& microbe 27, 841848 e843 (2020).

15. Song, W., Gui, M., Wang, X. \& Xiang, Y. Cryo-EM structure of the SARS coronavirus spike glycoprotein in complex with its host cell receptor ACE2. PLoS pathogens 14, e1007236 (2018).

16. Harcourt, J., et al. Severe Acute Respiratory Syndrome Coronavirus 2 from Patient with 2019 Novel Coronavirus Disease, United States. Emerg Infect Dis 26(2020).

17. Sims, A.C., et al. Release of severe acute respiratory syndrome coronavirus nuclear import block enhances host transcription in human lung cells. Journal of virology 87, 3885-3902 (2013). modulate global interferon stimulated gene induction through diverse mechanisms. Cytokine 59, 556-556 (2012).

482 19. Zhou, P., et al. A pneumonia outbreak associated with a new coronavirus of probable 483 bat origin. Nature 579, 270-273 (2020).

$48420 . \quad$ Imai, M., et al. Syrian hamsters as a small animal model for SARS-CoV-2 infection and countermeasure development. Proceedings of the National Academy of Sciences of the United States of America 117, 16587-16595 (2020).

488 diagnosis and vaccine evaluation. Nature communications 11, 4059 (2020). 
489

490

491

492

493

494

495

496

497

498

499

500

501

502

503

504

505

506

507

508

509

510

511

512

513

514

515

516

517

518

519

520

521

522

523

524

525

526

527

528

529

22. Davidson, A.D., et al. Characterisation of the transcriptome and proteome of SARS-CoV2 reveals a cell passage induced in-frame deletion of the furin-like cleavage site from the spike glycoprotein. Genome Med 12, 68 (2020).

23. Gao, Q., et al. Development of an inactivated vaccine candidate for SARS-CoV-2. Science 369, 77-81 (2020).

24. Hansen, J., et al. Studies in humanized mice and convalescent humans yield a SARSCoV-2 antibody cocktail. Science (2020).

25. Abe, M., et al. TMPRSS2 is an activating protease for respiratory parainfluenza viruses. Journal of virology 87, 11930-11935 (2013).

26. Roberts, A., et al. A mouse-adapted SARS-coronavirus causes disease and mortality in BALB/c mice. PLoS pathogens 3, e5 (2007).

27. Sims, A.C., et al. Release of severe acute respiratory syndrome coronavirus nuclear import block enhances host transcription in human lung cells. J Virol 87, 3885-3902 (2013).

28. Josset, L., et al. Cell host response to infection with novel human coronavirus EMC predicts potential antivirals and important differences with SARS coronavirus. MBio 4, e0016500113 (2013).

29. Sheahan, T., Rockx, B., Donaldson, E., Corti, D. \& Baric, R. Pathways of cross-species transmission of synthetically reconstructed zoonotic severe acute respiratory syndrome coronavirus. Journal of virology 82, 8721-8732 (2008).

30. Menachery, V.D., et al. Attenuation and restoration of severe acute respiratory syndrome coronavirus mutant lacking 2'-o-methyltransferase activity. Journal of virology 88, 4251-4264 (2014).

31. van Tol, S., et al. VAMP8 Contributes to the TRIM6-Mediated Type I Interferon Antiviral Response during West Nile Virus Infection. Journal of virology 94(2020).

32. Routh, A., Head, S.R., Ordoukhanian, P. \& Johnson, J.E. ClickSeq: Fragmentation-Free Next-Generation Sequencing via Click Ligation of Adaptors to Stochastically Terminated 3'Azido cDNAs. J Mol Biol 427, 2610-2616 (2015).

33. Chen, S., Zhou, Y., Chen, Y. \& Gu, J. fastp: an ultra-fast all-in-one FASTQ preprocessor. Bioinformatics 34, i884-i890 (2018).

34. Li, H., et al. The Sequence Alignment/Map format and SAMtools. Bioinformatics 25, 2078-2079 (2009).

35. Smith, T., Heger, A. \& Sudbery, I. UMI-tools: modeling sequencing errors in Unique Molecular Identifiers to improve quantification accuracy. Genome Res 27, 491-499 (2017).

36. Quinlan, A.R. \& Hall, I.M. BEDTools: a flexible suite of utilities for comparing genomic features. Bioinformatics 26, 841-842 (2010).

37. Waterhouse, A., et al. SWISS-MODEL: homology modelling of protein structures and complexes. Nucleic Acids Res 46, W296-W303 (2018).

38. Bienert, S., et al. The SWISS-MODEL Repository-new features and functionality. Nucleic Acids Res 45, D313-D319 (2017). 
531 Figure 1. Distinct replication, spike cleavage, and competition for $\triangle$ PRRA. A) Generation

532 of a SARS-CoV-2 mutant deleting the furin cleavage site insertion from the spike protein. B)

533 Structure of the SARS-CoV-2 spike trimer with a focus on the furin cleavage site (inset).

534 Modeled using the SARS-CoV-1 trimer structure (PDB 6ACD) (14), the WT SARS-CoV-2 trimer

535 (grey) with SARS-CoV-2 PRRA deletion mutant monomer overlay (red). The loop (inset), which

536 is unresolved on SARS-CoV-2 structures (AA 691-702), is shown in cyan on SARS-CoV-2 with

537 the PRRA sequence in blue. The loop region in the PRRA deletion mutant is shown in pink.

538 C) Viral titer from Vero E6 cells infected with WT SARS-CoV-2 (black) or $\triangle$ PRRA (blue) at MOI

$5390.01(\mathrm{~N}=3)$. D) Purified SARS-CoV, SARS-CoV-2 WT, and $\triangle \mathrm{PRRA}$ virions were probed with

540 anti-spike or anti-nucleocapsid antibody. Full length (FL), S1/S2 cleavage form, and S2'

541 annotated. E) Competition assay between SARS-CoV-2 WT (black) and $\triangle$ PRRA (blue) showing

542 RNA percentage based on quantitative RT-PCR at 50:50, 90:10, 10:90, 99:1, and 1:99 WT/

$543 \triangle \mathrm{PRRA}$ ratio ( $\mathrm{N}=3$ per group). $\mathrm{F}$ ) Viral titer from Calu3 2B4 cells infected with WT SARS-CoV-2

544 (black) or $\triangle$ PRRA (blue) at MOI $0.01(\mathrm{~N}=3)$. G) Purified SARS-CoV, SARS-CoV-2 WT, and

$545 \triangle$ PRRA virions were probed with anti-spike or anti-nucleocapsid antibody. Full length (FL),

546 S1/S2 cleavage form, and S2' annotated. P-values based on Student T-test and are marked as

547 indicated: ${ }^{*}<0.05^{* * *}<0.001$.

548 Figure 2. In vivo attenuation of $\triangle$ PRRA mutant. A) Primary SARS-CoV-2 challenge

549 schematic. Two groups of male hamsters $(\mathrm{N}=4)$ were challenged with $10^{5}$ plaque forming units

550 of either SARS-CoV-2 WT or $\triangle$ PRRA mutant and evaluated over a 28 day time course for B)

551 weight loss, C) disease score, D) viral titer from nasal wash, and E) viral RNA from oral swabs.

552 F) Schematic for rechallenge of previously infected hamsters. Twenty eight DPI, hamsters from

553 SARS-CoV-2 WT and $\triangle$ PRRA were rechallenged with $10^{5}$ PFU of SARS-CoV-2 WT and

554 evaluated for $\mathrm{G}$ ) weight loss, $\mathrm{H}$ ) disease score, I) viral titer from nasal wash, and E) viral RNA 
555 from oral swabs. P-values based on Student T-test and are marked as indicated: * $<0.05$

$556^{* *}<0.01^{* * *}<0.001$.

557 Figure 3. Antibody neutralization of $\triangle \mathrm{PRRA}$ mutant. A) Schematic for SARS-CoV-2 $\triangle P R R A$

558 reporter virus expressing $\mathrm{mNeonGreen}(\mathrm{mNG})$ gene in place of ORF7 equivalent to previously

559 described WT SARS-CoV-2 mNG virus ${ }^{21}$. B) Plaque reduction neutralization $\left(\mathrm{PRNT}_{50}\right)$ values

560 as measured by changes to $\mathrm{mNG}$ expression. PRNT 50 values plotted as Log (1/serum dilution)

561 with $\triangle P R R A$ on $Y$ axis and WT-SARS-CoV-2. C-E) Representative curves from C) low, D)

562 intermediate, and E) high neutralizing COVID-19 patient sera. F-H) Neutralization curves from

563 mAB-1 $(F), m A B-2(G)$, and mAB-3 $(H), N=3$. 

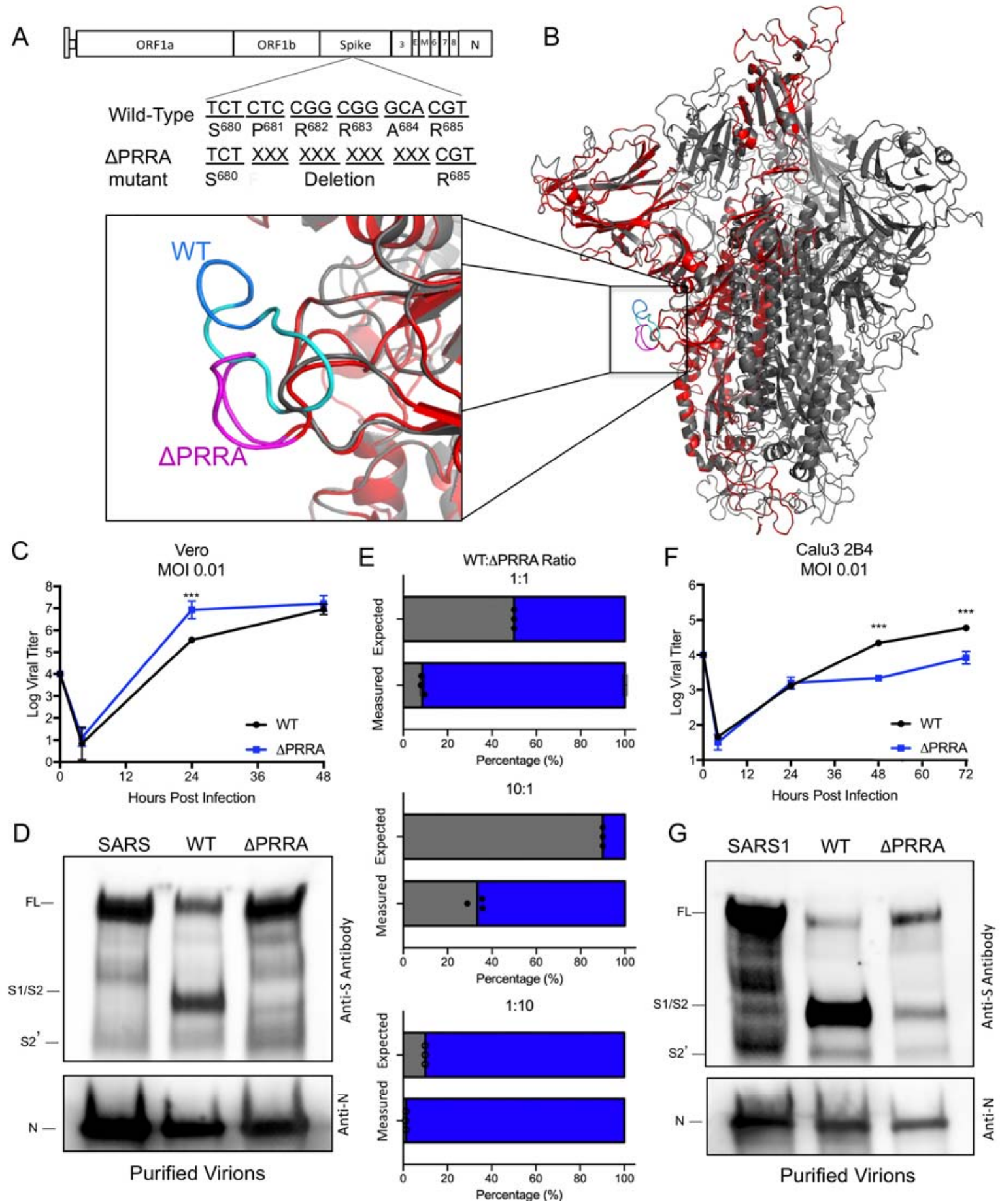

564 Figure 1. Distinct replication, spike cleavage, and competition for $\triangle P R R A$. A) Generation of a SARS-CoV-2 mutant deleting the furin cleavage site insertion from the spike protein. B) Structure of the SARS-CoV-2 spike trimer with a focus on the furin cleavage site (inset). Modeled using the SARS-CoV-1 trimer structure (PDB 6ACD) (14), the WT SARS-CoV-2 trimer (grey) with SARS-CoV-2 PRRA deletion mutant monomer overlay (red). The loop (inset), which is unresolved on SARS-CoV-2 structures (AA 691-702), is shown in cyan on SARS-CoV-2 with the PRRA sequence in blue. The loop region in the PRRA deletion mutant is shown in pink. $0.01(\mathrm{~N}=3)$. D) Purified SARS-CoV, SARS-CoV-2 WT, and $\triangle \mathrm{PRRA}$ virions were probed with 
574 anti-spike or anti-nucleocapsid antibody. Full length (FL), S1/S2 cleavage form, and S2'

575 annotated. E) Competition assay between SARS-CoV-2 WT (black) and $\triangle$ PRRA (blue) showing

576 RNA percentage based on quantitative RT-PCR at 50:50, 90:10, 10:90, 99:1, and 1:99 WT/

$577 \triangle \mathrm{PRRA}$ ratio ( $\mathrm{N}=3$ per group). $\mathrm{F}$ ) Viral titer from Calu3 2B4 cells infected with WT SARS-CoV-2

578 (black) or $\triangle$ PRRA (blue) at MOI $0.01(\mathrm{~N}=3)$. G) Purified SARS-CoV, SARS-CoV-2 WT, and

$579 \triangle \mathrm{PRRA}$ virions were probed with anti-spike or anti-nucleocapsid antibody. Full length (FL),

580 S1/S2 cleavage form, and S2' annotated. P-values based on Student T-test and are marked as

581 indicated: ${ }^{*}<0.05^{* * *}<0.001$. 
A

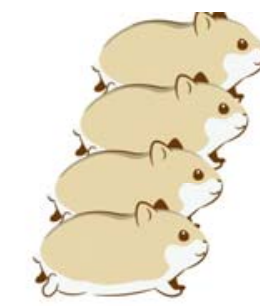

Two Groups of Four Male Hamsters

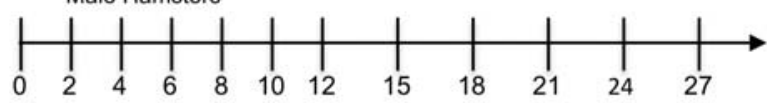

C

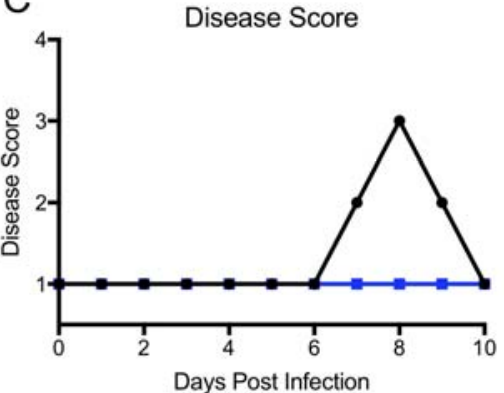

$\mathrm{F}$

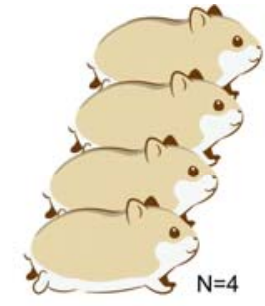

Previously Infected with WT or $\triangle P R R A$

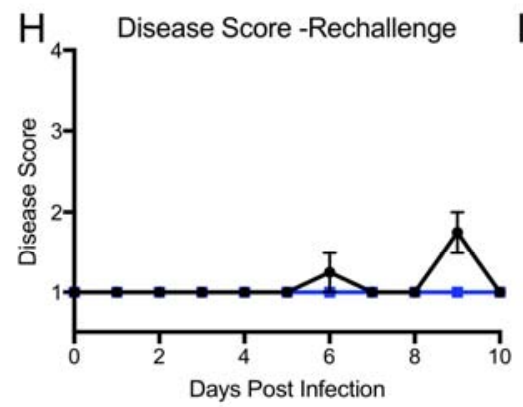

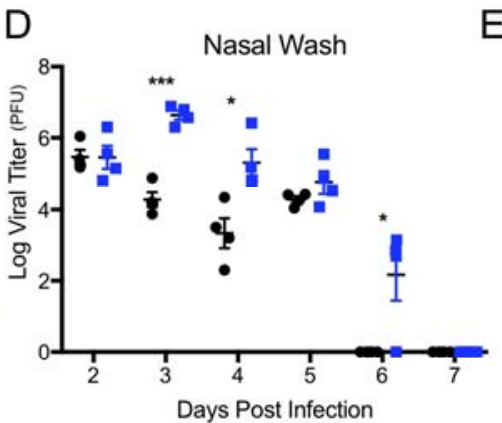

B

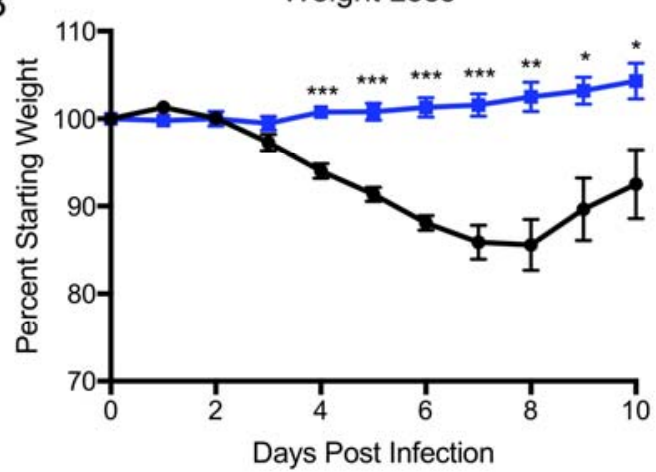

E

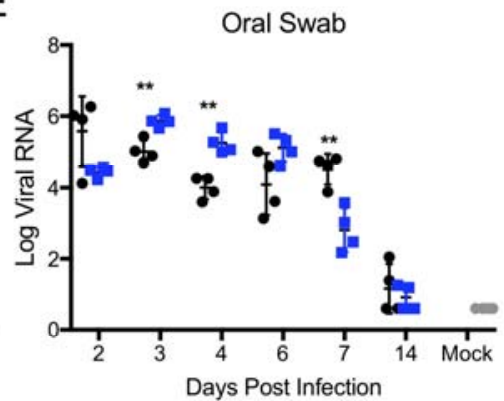

G

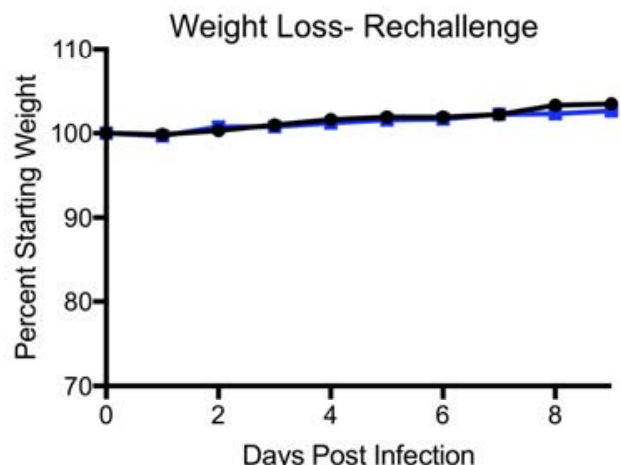

Days Post Infection

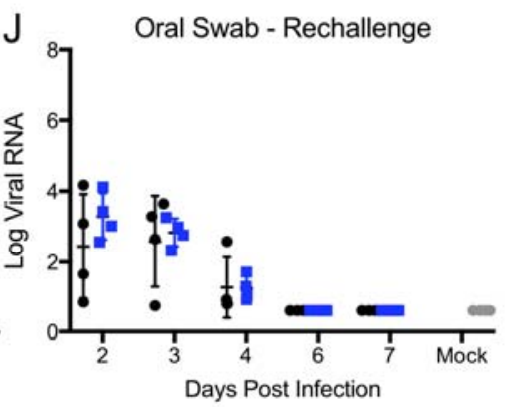

Figure 2. In vivo attenuation of $\triangle$ PRRA mutant. A) Primary SARS-CoV-2 challenge schematic. Two groups of male hamsters $(\mathrm{N}=4)$ were challenged with $10^{5}$ plaque forming units of either SARS-CoV-2 WT or $\triangle$ PRRA mutant and evaluated over a 28 day time course for B) weight loss, C) disease score, D) viral titer from nasal wash, and E) viral RNA from oral swabs. F) Schematic for rechallenge of previously infected hamsters. Twenty eight DPI, hamsters from SARS-CoV-2 WT and $\triangle$ PRRA were rechallenged with $10^{5}$ PFU of SARS-CoV-2 WT and evaluated for G) weight loss, H) disease score, I) viral titer from nasal wash, and E) viral RNA from oral swabs. P-values based on Student T-test and are marked as indicated: ${ }^{*}<0.05^{* *}<0.01^{* * *}<0.001$. 
A

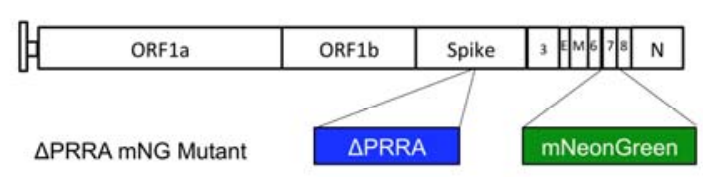

B
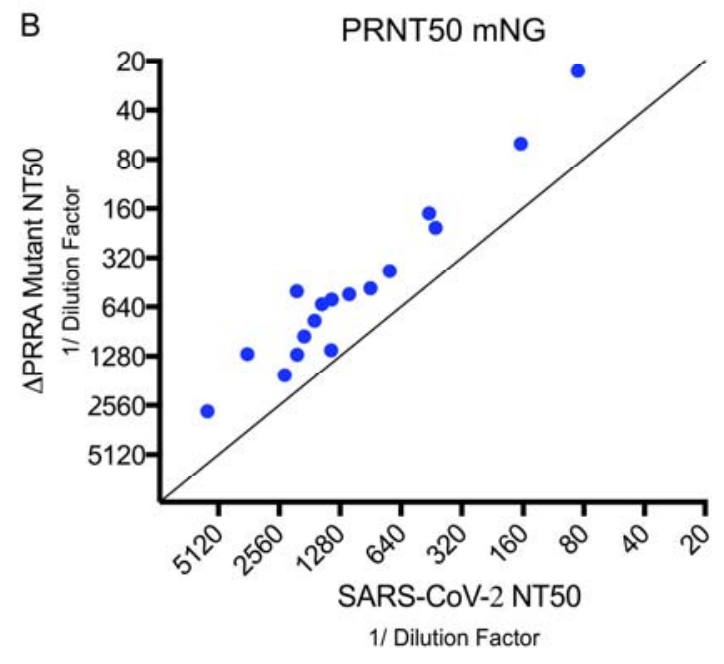

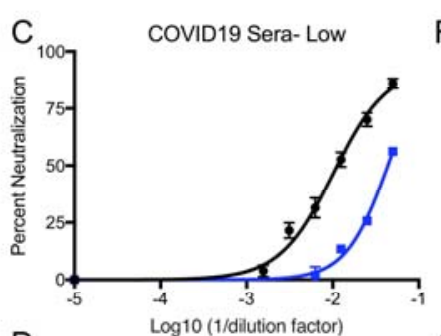

D $\quad$ Log $10(1 /$ dilution factor $)$
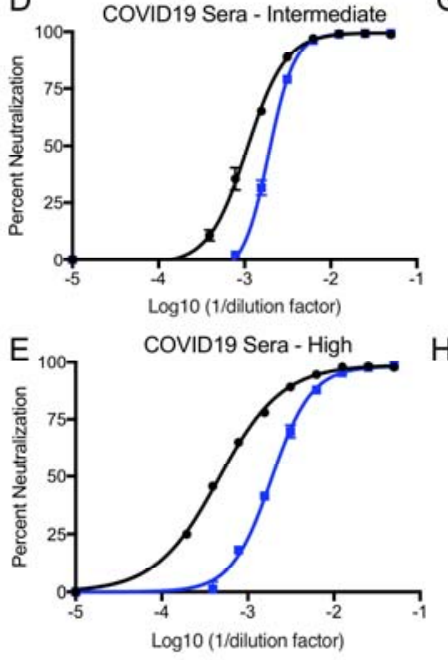

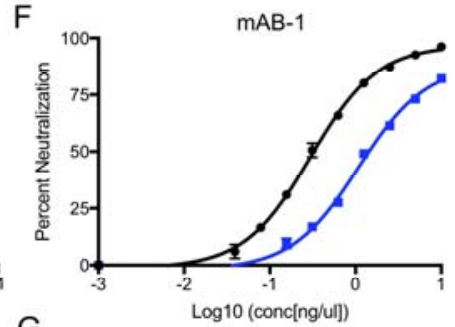

$G \quad \mathrm{mAB}-2$
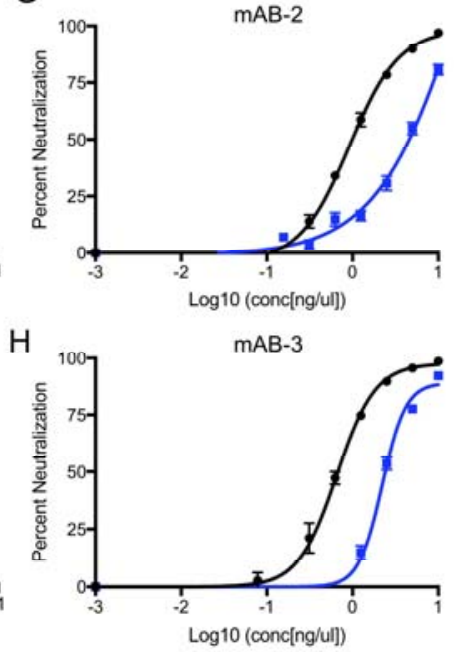

Figure 3. Antibody neutralization of $\triangle$ PRRA mutant. A) Schematic for SARS-CoV-2 $\triangle P R R A$ reporter virus expressing $\mathrm{mNeonGreen} \mathrm{(mNG)} \mathrm{gene} \mathrm{in} \mathrm{place} \mathrm{of} \mathrm{ORF7} \mathrm{equivalent} \mathrm{to} \mathrm{previously} \mathrm{described} \mathrm{WT}$ SARS-CoV-2 mNG virus ${ }^{14}$. B) Plaque reduction neutralization (PRNT ${ }_{50}$ ) values as measured by changes to $\mathrm{mNG}$ expression. $\mathrm{PRNT}_{50}$ values plotted as Log (1/serum dilution) with $\triangle \mathrm{PRRA}$ on $\mathrm{Y}$ axis and WTSARS-CoV-2 on the $X$ axis. $C-E)$ Representative curves from $C$ ) low, $D$ ) intermediate, and $E$ ) high neutralizing COVID-19 patient sera. F-H) Neutralization curves from mAB-1 (F), mAB-2 (G), and mAB-3 
A
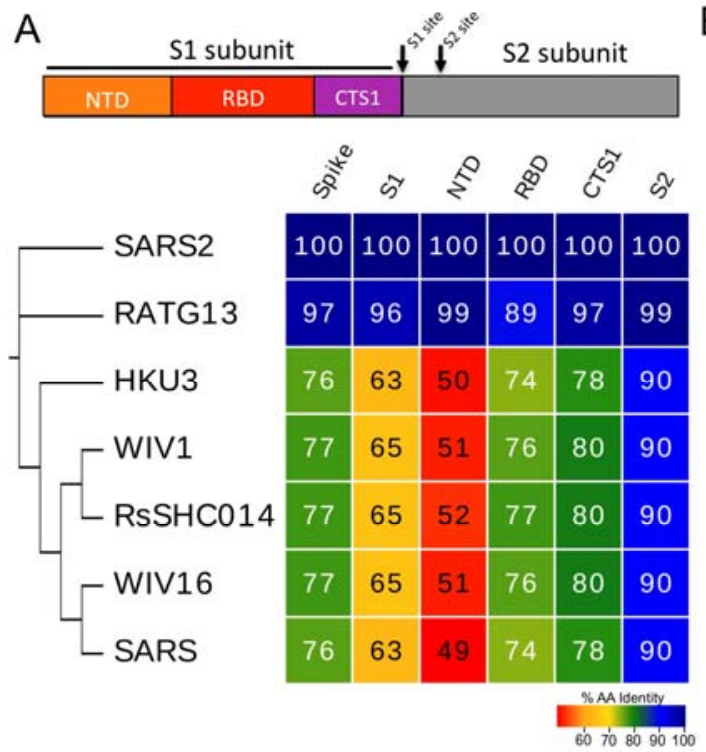

B \begin{tabular}{l|lllllll|l|l|l|l|l|l|llll} 
SARS-2 & S & Y & Q & T & Q & T & N & S & P & R & R & A & R & S & V \\
RATG13 & S & Y & Q & T & Q & T & N & S & - & - & - & - & R & S & V \\
HKU3 & S & Y & H & T & A & S & V & L & - & - & - & - & - & R & S & T \\
WIV1 & S & Y & H & T & V & S & S & L & - & - & - & - & R & S & T \\
SHC014 & S & Y & H & T & V & S & S & L & - & - & - & - & - & R & S & T \\
WIV16 & S & Y & H & T & V & S & S & L & - & - & - & - & - & R & S & T \\
SARS & S & Y & H & T & V & S & S & L & - & - & - & - & R & S & T
\end{tabular}

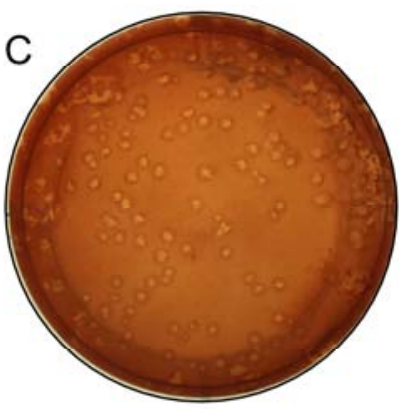

WT

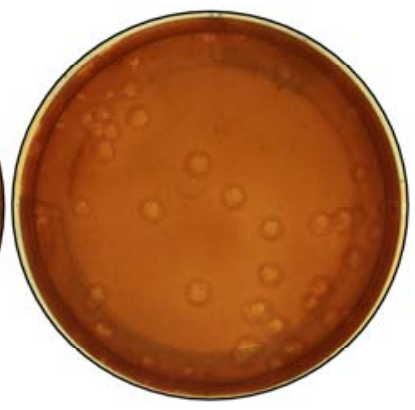

$\triangle P R R A$

602 S. Figure 1. Furin cleavage site in SARS-CoV-2 spike. A) Diagram of the coronavirus spike protein domains and cleavage sites. The sequences of the indicated group 2B coronaviruses were aligned according to the bounds of total spike, S1, N-terminal domain (NTD), Receptor binding domain (RBD), and C-terminal of S1 (CTS1) and S2. Sequence identities were extracted from the alignments, and a heatmap of sequence identity was constructed using EvolView (www.evolgenius.info/evolview) with SARS-CoV-2 WA1 as the reference sequence. B) Alignment of the furin cleavage site of SARS-CoV-2 and the corresponding amino acids identities found closely related group $2 \mathrm{~B}$ CoVs. The PRRA insertion is unique to SARS-CoV-2 $\quad$ C) Representative plaque morphology of WT and $\triangle$ PRRA SARS-CoV-2. 


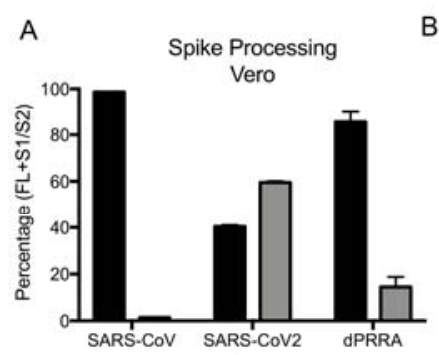

D

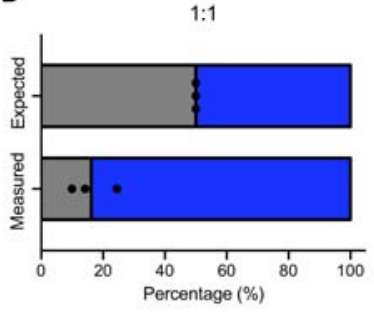

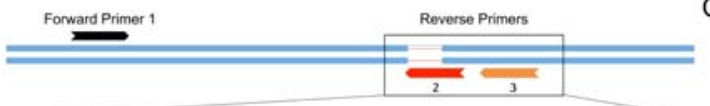
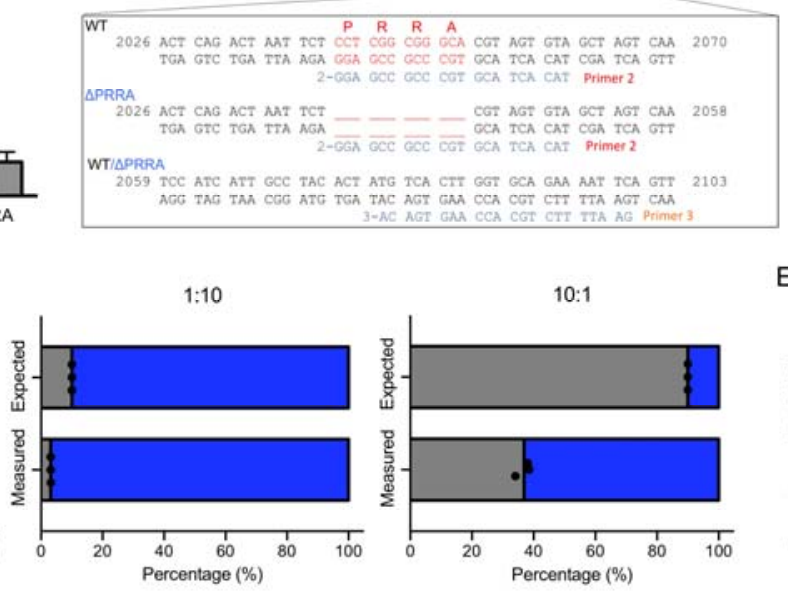

10:1

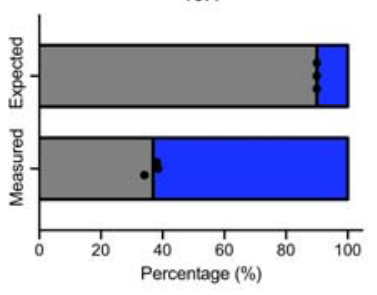

C

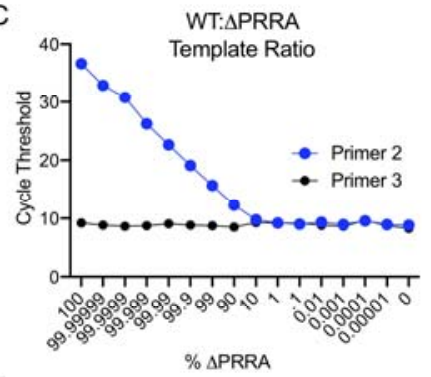

$\mathrm{E}$

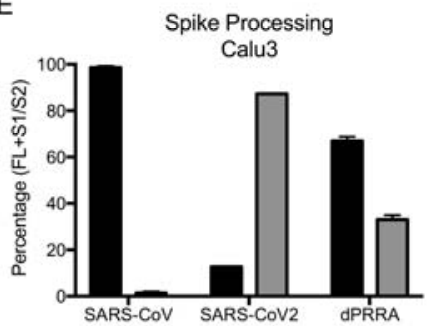

612 S. Figure 2. $\triangle$ PRRA mutant processing and competition with WT. A) Quantitation by densitometry of the full-length spike (Black) and S1/S2 cleavage form (Gray) from distinct western blot experiments in Vero E6 cells $(n=2)$. B) Schematic of quantitative RT-PCR approach to detect deletion of the furin cleavage site. C) Primer curve validation with mixed WT to $\triangle$ PRRA plasmid ratio showing level of sensitivity. D) Deep sequencing results from $\triangle P R R A$ and WT competition assays based on percentage of total reads in that region ( $\mathrm{N}=3$ ). E) Quantitation by densitometry of the full-length spike (Black) and $\mathrm{S} 1 / \mathrm{S} 2$ cleavage form (Gray) from distinct western blot experiments from Calu3 ( $n=2)$. 

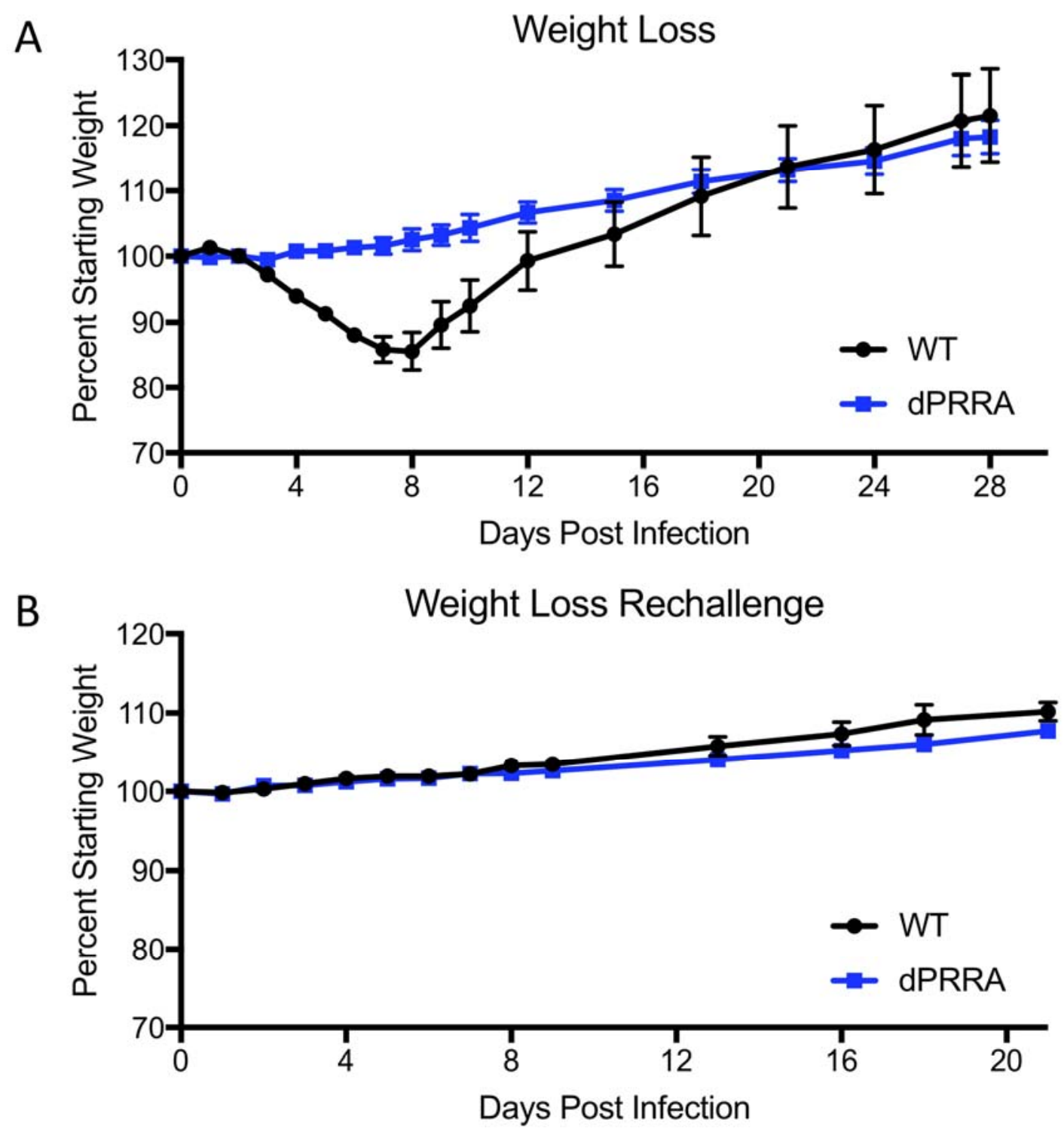

621 S. Figure 3. In vivo attenuation of $\triangle$ PRRA mutant. A) Weight loss following primary WT and $\triangle P R R A$ 622 mutant SARS-CoV-2 challenge ( $\mathrm{N}=4$ per group). B) Weight loss following rechallenge of WT and $\triangle P R R A$ 623 mutant infected mice with WT SARS-CoV-2 ( $\mathrm{N}=4$ per group). 